\title{
Case series analysis of post-brachytherapy prostate edema and its relevance to post-implant dosimetry. Post-implant prostate edema and dosimetry
}

\author{
Ajay Tejwani, MD, MPH', Eva Bieniek, MD², Lindsay Puckett, BS', Amir Lavaf, MD', Adel Guirguis, MS', Aaron Bennish, PhD', \\ Hani Ashamalla, MD'.3 \\ INew York Methodist Hospital, Brooklyn, New York, 2 Cancer Center of Hawaii, Weinberg Medical, Pavilion, Honolulu, Hawaii, 3 Weill Medical \\ College of Cornell University. New York, USA
}

\begin{abstract}
Purpose: We evaluated the post-operative pattern of prostate volume (PV) changes following prostate brachytherapy $(\mathrm{PB})$ and analyzed variables which affect swelling.

Material and methods: Twenty-nine patients treated with brachytherapy (14) or combined brachytherapy and external beam radiotherapy modality (15) underwent pre- and post-implant computed tomography (CT). Prostate volume measurements were done on post-operative days 1, 9, 30, and 60. An observer performed 139 prostate volume (PV) measurements. We analyzed the influence of pre-implant PV, number of needles and insertion attempts, number and activity of seeds, Gleason score, use of hormonal therapy and external beam radiation therapy on the extent of edema. We computed a volume correction factor (CF) to account for dosimetric changes between day 1 and day 30 . Using the calculated CF, the dose received by $90 \%\left(\mathrm{D}_{90}\right)$ of the prostate on day $30\left(\mathrm{D}_{90}\right.$ Day 30$)$ was obtained by dividing day $1\left(\mathrm{D}_{90} \mathrm{Day} 1\right)$ by the CF.

Results: The mean PV recorded on post-operative day 1 was $67.7 \mathrm{~cm}^{3}, 18.8 \mathrm{~cm}^{3}$ greater than average pre-op value $\left(\mathrm{SD} 15.6 \mathrm{~cm}^{3}\right)$. Swelling returned to pre-implant volume by day 30 . Seed activity, treatment modality, and Gleason score were significant variables. The calculated $\mathrm{CF}$ was 0.76 . After assessment using the $\mathrm{CF}$, the mean difference between estimated and actual $\mathrm{D}_{90}$ Day30 was not significant.

Conclusions: We observed maximum prostate size on post-operative day 1, returning to pre-implant volume by day 30 . This suggests that post-implant dosimetry should be obtained on or after post-operative day 30 . If necessary, day 30 dosimetry can be estimated by dividing $\mathrm{D}_{90}$ Day1 by a correction factor of 0.76 .
\end{abstract}

J Contemp Brachyther 2012; 4, 2: 75-80 DOI: $10.5114 / \mathrm{jcb} .2012 .29363$

Key words: prostate brachytherapy, post-implant dosimetry, computed brachytherapy.

\section{Purpose}

Prostate cancer is the most common non-cutaneous cancer in men. According to the most recent SEER data, 217730 men are estimated to have been diagnosed with prostate cancer in 2010 resulting in 32050 prostate cancer related deaths [1]. Transperineal interstitial prostate implant, either by itself or in combination with external beam radiotherapy (EBRT), is a well accepted treatment modality for men with low and intermediate risk of disease. Data have shown that appropriate patient populations treated with prostate brachytherapy have improved biochemical relapse-free survival (bRFS) as compared to patients treated with radical prostatectomy (RP) and EBRT [2-5]. Computed tomography (CT) of the pelvis and transrectal prostate ultrasound (TRUS) are central to the evaluation and management of patients with prostate cancer. Pelvic
$\mathrm{CT}$ is often recommended for staging patients with a risk of pelvic lymph node involvement greater than $20 \%$ [6]. In addition to being a part of the staging work-up, CT is used in determining post-implantation seed placement and in calculating the dose delivered to the prostate and to the structures at risk [7]. TRUS is generally used to assess preimplant prostate volumes as well as intra-operative placement of radioactive sources. Trauma from brachytherapy seed implantation is associated with a significant increase in prostate size which may affect post-procedure dose volume calculations. If the post-implant CT used for dosimetry calculations is performed shortly after the procedure, the calculated delivery dose may appear suboptimal. Waterman et al. performed pre- and post-procedure serial CT scans on 10 patients who underwent either palladium103 or iodine- 125 prostate seed brachytherapy. The study showed a mean 1.52-fold increase in prostate volumes, rang- 
ing from 1.33 to 1.96. Edema was most notable on the first day after the procedure and resolved exponentially with time. Mean edema half-life was 9.3 days (range 4 to 25 days). Edema had a significant impact on 7 of 10 patients' postimplant dosimetry [8]. Other authors have examined postimplantation edema's dosimetric effects using CT, ultrasound, and magnetic resonance imaging (MRI); many have proposed methods for the correction of dosimetric discrepancy due to prostate swelling [9-21]. Current guidelines from the American Association of Physicists in Medicine (AAPM) advocate that in I-125 seed implantation, dosimetry should be calculated 1 month ( \pm 1 week) after implantation to allow for resolution of edema [22]. Despite its limitations, CT continues to be the most commonly used imaging modality for obtaining post-implant dosimetric calculations within the community setting. In addition to the debate over imaging modality, the factors which influence edema are not well agreed upon by the research community. Thus, further research is warranted to elucidate relevant factors for post-implant prostate edema and resultant effects on dosimetry.

This study was designed to track the pattern of edema resolution over time, evaluate the extent of prostate swelling following I-125 seed implantation, and examine the effects of several treatment-related variables on mag nitude of edema. We analyzed the effect of prostate volume changes on post-implant dosimetry and proposed an uncomplicated correction factor to estimate the change in dosimetry between day 1 and day 30 .

\section{Material and methods}

\section{Patient enrollment}

This study was approved by the Institutional Review Board. Between February 2006 and December 2006 we enrolled 37 patients. Eight patients did not have sufficient fol-

Table 1. Summary of patient and treatment characteristics

\begin{tabular}{|c|c|}
\hline Average Gleason score & 6.6 \\
\hline Average initial PSA (ng/mL) & 6.4 \\
\hline Average number of needles & 15.4 \\
\hline $\begin{array}{l}\text { Average number of needle } \\
\text { insertion attempts }\end{array}$ & 20.2 \\
\hline Number of seeds & 65.4 \\
\hline Average activity (mCi) & 0.4 \\
\hline Hormone ablation therapy & $\begin{array}{l}\text { Yes }-5 \\
\text { No }-24\end{array}$ \\
\hline Number receiving EBRT & $\begin{array}{c}15(52 \%) \\
\text { Group } 1 \text { (EBRT followed } \\
\text { by implant) }-7 \\
\text { Group } 2 \text { (Implant followed } \\
\text { by EBRT) }-8\end{array}$ \\
\hline Average EBRT dose (Gy) & 47.6 \\
\hline
\end{tabular}

low-up, so 29 were included in the preliminary analysis. Patients received either brachytherapy alone or a combined modality approach with PB and EBRT in a non-randomized fashion based on the clinical stage, Gleason score, and prostate-specific antigen (PSA) level. Patients with a Gleason score of $\leq 6$ and PSA of $\leq 10$ with no other significant risk factors were placed in brachytherapy alone. For patients receiving EBRT, sequencing of brachytherapy and EBRT was based on the patient's preference and schedule availability. Fourteen patients were treated with brachytherapy alone (Group 0) and fifteen patients received a combination treatment. In the combined modality group, seven patients underwent prostate implant immediately following completion of EBRT to 50 Gy (Group 1). The remaining eight received EBRT four to five weeks after the procedure (Group 2). In addition to radiation, five patients from the three groups received hormonal therapy three months prior to receiving brachytherapy. Patients in groups 1 and 2 were statistically similar in their averaged Gleason and PSA. Patient characteristics are listed in Table 1 and the general management schema is illustrated in Fig. 1.

\section{Prostate volume measurements}

Pelvic CT with urethrogram was used for all prostate volume (PV) measurements. The urethrogram was performed by inserting the tip of a syringe containing a radio-opaque contrast material into the urethral meatus and injecting $10 \mathrm{cc}$ of contrast into the urethra. The prostate apex was marked $1 \mathrm{~cm}$ above the urethral beak [21]. The prostate was outlined on each $3 \mathrm{~mm}$ CT slice by a single observer and prostate volume was computed automatically. All patients had a preimplant prostate CT with urethrogram prior to the procedure. Post-brachytherapy serial PV measurements were done on post-op day 1 (PV1), day 9 (PV9), day 30 (PV30), and day 60 (PV60).

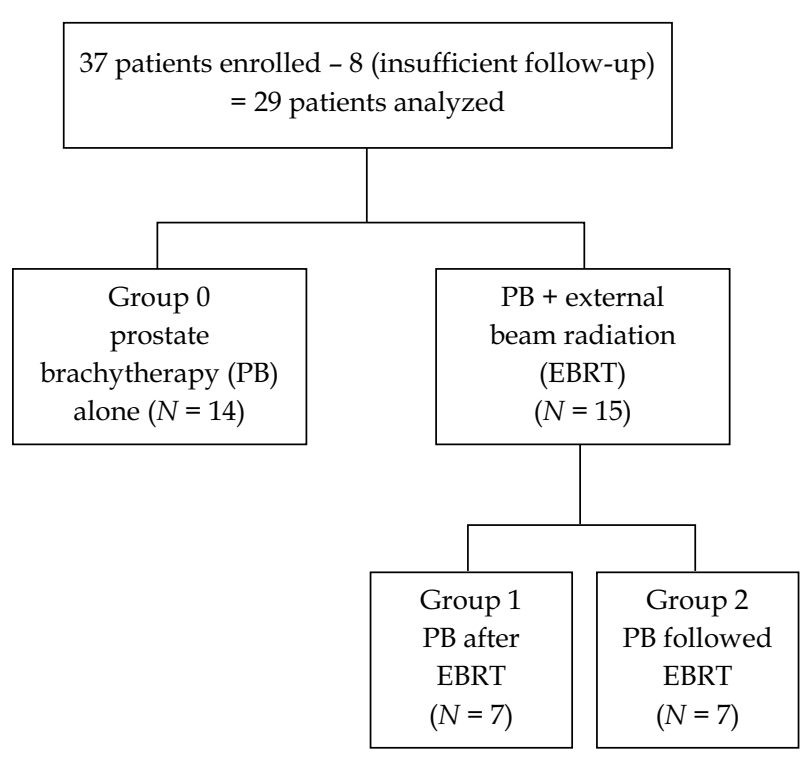

$P B$ - prostate brachytherapy, EBRT - external beam radiation

Fig. 1. Overview of treatment 


\section{Determinants of PV change}

The influence of several variables on post-implantation PV was examined using univariate analysis. Non-parametric tests were used to compare the serial data. The variables included in the analysis were the following: 1) pre-implant prostate volume; 2) number of needles used;3) number of needle insertion attempts; 4) number of seeds; 5) seed activity; 6) Gleason score; 7) hormonal ablation therapy (HAT); 8) EBRT.

\section{Dosimetric calculations}

The quality of an implant was assessed by determining the dose received by $90 \%$ of the prostate $\left(\mathrm{D}_{90}\right)$. $\mathrm{D}_{90}$ Day1 and $\mathrm{D}_{90}$ Day30 were computed using prostate contours delineated from scans taken on post-op day 1 and day 30, respectively.

A volume correction factor $(\mathrm{CF})$ was calculated for each patient as a ratio of their prostate volume measured on day $30\left(\mathrm{PV}_{30}\right)$ to that on day $1\left(\mathrm{PV}_{1}\right)$.

$\mathrm{CF}=\mathrm{PV}_{30} / \mathrm{PV}_{1}$

The estimated $\mathrm{D}_{90}$ Day30 $\left(\mathrm{eD}_{90}\right.$ Day30) and the average estimated $\mathrm{D}_{90}$ Day30 (ED ${ }_{90}$ Day30) were calculated by dividing $\mathrm{D}_{90}$ Day1 by the individual's $C F$ as well as the study's mean CF as illustrated in equations 2 and 3, respectively.

Estimated $\mathrm{D}_{90}$ on Day 30 (per individual): eD ${ }_{90}$ Day30 $=\mathrm{D}_{90}$ Day1/individual CF

Average Estimated D90 on Day 30 (per individual): $\mathrm{ED}_{90}$ Day30 $=\mathrm{D}_{90}$ Day $1 /$ average $\mathrm{CF}$

$\mathrm{D}_{90}$ Day1 and $\mathrm{ED}_{90}$ Day30 (from equation 3) were compared with $\mathrm{eD}_{90}$ Day30 (from equation 2) using the Sign test.

\section{Statistical considerations}

This is an exploratory, hypothesis-generating study to find a model which simulates the dose received by $90 \%$ of the prostate (D90) on Day 30. Data were summarized and non-parametric statistical tests, sign and Wilcoxon rank-sum tests, were conducted to compare the relevant variables.

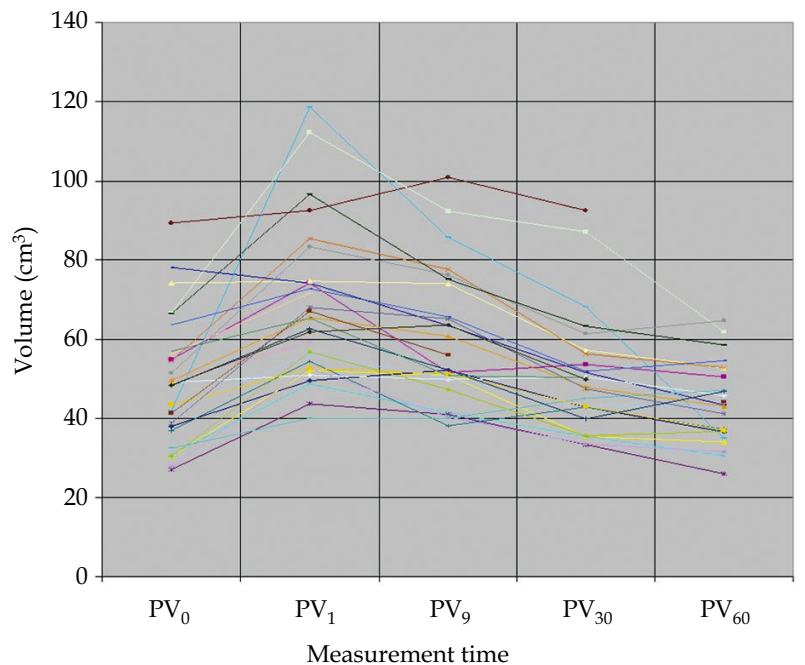

PV - prostate volume

Fig. 2. Pattern of prostate volume changes over time in individual patients

Univariate regression analysis was performed to test the relation between different variables and the change in prostate volume one day post-implantation. All reported $P$ values are two-sided and values of 0.05 or lower are considered to indicate statistical significance.

\section{Results}

\section{Pattern of prostate volume change over time}

A total of 139 PV measurements were obtained. Prostate size increased by an average factor of $1.44 \pm 0.37$ at the time of maximum swelling, which occurred in the immediate post-operative period. The mean prostate volume recorded on post-operative day $1\left(67.7 \mathrm{~cm}^{3}\right)$ was $18.8 \mathrm{~cm}^{3}$ greater than the average pre-op value of $48.9 \mathrm{~cm}^{3}$ (SD 15.6). Prostate swelling gradually subsided over time. At one month post-implantation, the difference between pre- and post-operative prostate volumes was no longer significant (Fig. 2, Tables 2 and 3).

Table 2. Mean change in PV and IPSS over time

\begin{tabular}{lccccc} 
& Day 0 & Day 1 & Day 9 & Day 30 & Day 60 \\
\hline Mean PV $\left(\mathrm{cm}^{3}\right) \pm$ standard deviation & $48.9 \pm 15.7$ & $67.7 \pm 19.2$ & $59.8 \pm 16.7$ & $50.8 \pm 14.7$ & $44 \pm 9.9$ \\
\hline Ratio of mean $\mathrm{PV}_{\mathrm{x}}$ to baseline $\left(\mathrm{PV}_{0}\right)$ & 1 & 1.38 & 1.2 & 1.03 & 0.89
\end{tabular}

$P V$ - prostate volume, $P V_{x}-P V$ on day

Table 3. Mean difference in prostate volume change from days 0, 1, 9, and 30 to later measurement

\begin{tabular}{lcccc} 
& $\mathrm{PV}_{1}$ & $\mathrm{PV}_{9}$ & $\mathrm{PV}_{30}$ & $\mathrm{PV}_{60}$ \\
\hline $\mathrm{PV}_{0}$ & $+18.8 \pm 15.6(p<0.0001)$ & $+11.2 \pm 11.9(p=0.0003)$ & $+1.86 \pm 10.7(\mathrm{NS})$ & $-2.7 \pm 9.8(\mathrm{NS})$ \\
\hline $\mathrm{PV}_{1}$ & - & $-8.1 \pm 9.0(p<0.0001)$ & $-17.2 \pm 11.1(p<0.0001)$ & $-22.9 \pm 16.6(p<0.0001)$ \\
\hline $\mathrm{PV}_{9}$ & - & - & $-9.2 \pm 7.1(p=0.0005)$ & $-14.3 \pm 11.5(p<0.0001)$ \\
\hline $\mathrm{PV}_{30}$ & - & - & - & $-5.1 \pm 8.6(p=0.01)$
\end{tabular}

PV - prostate volume, PV $-P V$ on day $x, N S$ - not statistically significant; "+" indicates increase and "-" decrease with respect to the PV on days 0 , 1, 9, or 30, as indicated in each row 
Figure 3 is a regression fit graph representing the relationship between the pre and post-implant prostate volumes. One can estimate a PV on a given post-operative day by plotting the pre-implant value on the $\mathrm{x}$-axis and tracing the value to the regression line, and reading off the expected value.

\section{Determinants of prostate swelling}

We examined several factors and their influence on the degree of prostate swelling. Using logistic regression analysis, we assessed which variables lead to a greater than one third increase of the initial prostate volume one day after the implantation. Our results indicate that seed activity ( $\leq 0.4$ vs. $>0.4 \mathrm{mCi}$ ), treatment modality (brachytherapy alone vs. combined modality), and Gleason score ( $\leq 6$ vs. $>6$ ) were statistically significant variables with respect to prostate edema ( $p$ values $=0.02,0.02$, and 0.046 respectively).

There was a tendency for higher seed activity $(>0.4 \mathrm{mCi})$, low Gleason score $(\leq 6)$, and treatment with brachytherapy alone to be associated with a greater change in prostate volume. Within our study, the low-risk group patients met all three of these conditions. Patients with low-risk disease (as determined by Gleason and PSA) were implanted with

A

Regression line to show

the changes in prostate volumes between day 1 and day 0

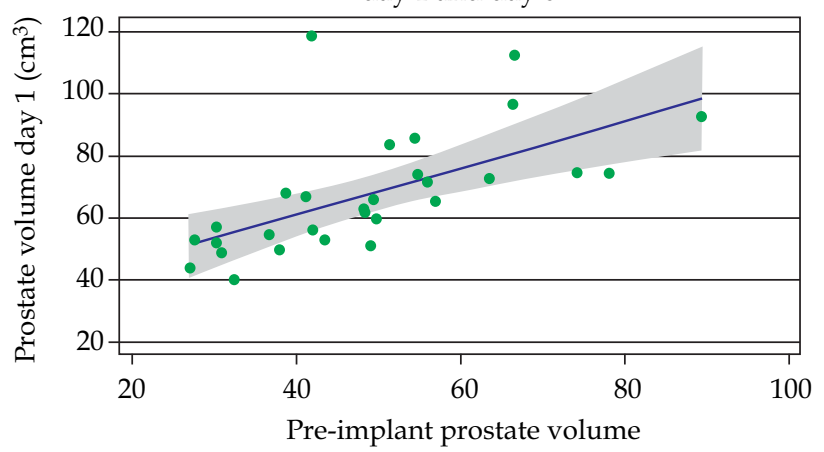

C

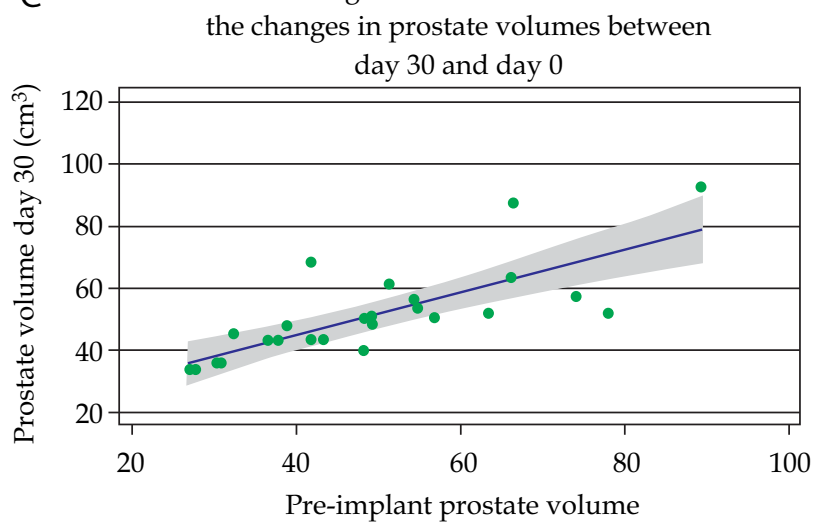

higher activity seeds and were found to have an average prostate swelling of $24.8 \mathrm{~cm}^{3}$, compared to only a $13.1 \mathrm{~cm}^{3}$ volume increase in patients implanted with lower activity seeds $(p$ value $=0.02)$. None of the remaining factors such as pre-implant prostate volume, number of needles and insertion attempts, number and activity of seeds, use of hormonal therapy, or the sequencing of EBRT were found to be significantly correlated with the degree of prostate edema one day after the procedure.

\section{Influence of prostate swelling on dosimetry}

The individual's volume correction factor was calculated as a ratio of PV30 to PV1 as described by equation 1 $(\mathrm{CF}=\mathrm{PV} 30 / \mathrm{PV} 1)$. The mean volume correction factor was determined to be $0.76 \pm 0.21$. For each patient, an estimated $\mathrm{D}_{90}$ Day30 value ( $\mathrm{eD}_{90}$ Day30) was calculated using the individual's CF and the mean $\mathrm{CF}$, as illustrated in equations 2 and 3 , respectively.

The discrepancy between the day 1 and day 30 dosimetry was significant with the calculated ratio between day 1 and day 30 dosimetry (Actual $\mathrm{D}_{90}$ Day1/Actual $\mathrm{D}_{90}$ Day 30 ) found to be $0.7117(p=0.0078)$ (Table 4). Using our proposed

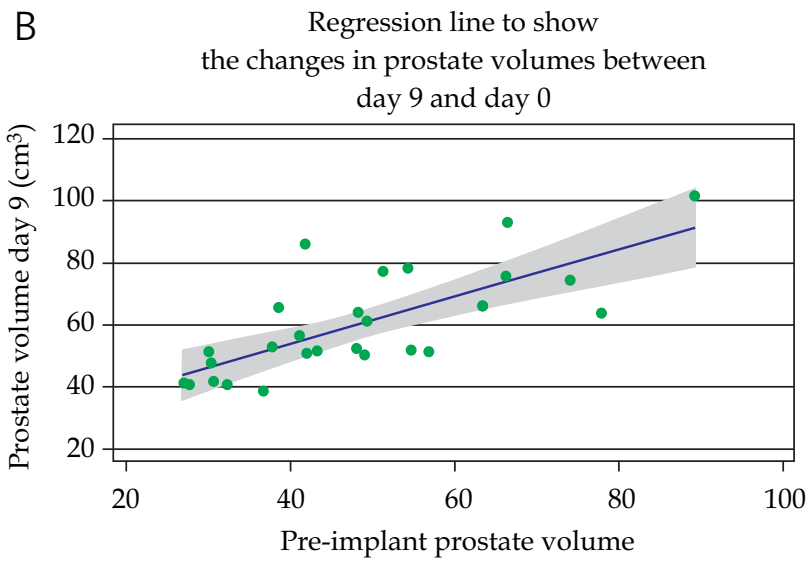

D Regression line to show the changes in prostate volumes between day 60 and day 0

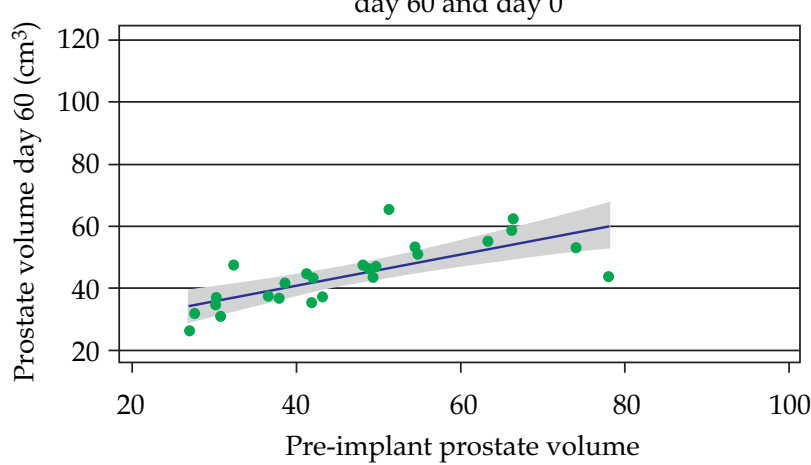

$$
\begin{aligned}
& \square \text { 95\% Cl } \\
& \square \text { Fitted values } \\
& \text { - Prostate volume day }
\end{aligned}
$$

Fig. 3. Correlation between pre- and post-implant prostate volume on post-operative (A) day 1, (B) day 9, (C) day 30, and (D) day 60 
$\mathrm{CF}$, we next compared the mean estimated $\mathrm{D}_{90}\left(\mathrm{ED}_{90}\right.$ Day30) to the actual $\mathrm{D}_{90}$ for day $30\left(\mathrm{D}_{90} \mathrm{Day} 30\right)$. This yielded a ratio of $0.9365(p=1.000$, NS) for all patients. Our CF was therefore successful in accounting for the effects of edema on day 1 dosimetry.

\section{Discussion}

The pattern of edema resolution shown in our study is consistent with previously published reports. The timing of PV measurement was chosen based on published data regarding edema resolution pattern. The preponderance of data suggests that edema half-life is $8-10$ days on average, returning to pre-implant size by day 30 [8-21]. Our results pertaining to the prostate edema resolution pattern are similar to previously published data. The results are also consistent with current AAPM recommendations which suggest the use of post-implantation $\mathrm{CT}$ at day 30 to allow for resolution of edema [22].

Waterman et al. also determined the edema half-life to be 9.3 days (range: 4 to 25 days). Edema had a significant impact on 7 of 10 patients' post-implant dosimetry [8]. The post-implant dosimetry was based on CT-based contours, as in our study. In our study, we found the edema half-life to be 17.3 days. Waterman found a positive correlation between seed spacing and PV, but did not appreciate any correlation between intra-operative variables such as number of needles, radioisotope, number of seeds implanted, and the total source strength. Similarly, our study found no appreciable correlation between number of needles and number of seeds. In another study, Prestidge et al. examined 19 patients treated with either iodine- 125 or palladium-103 implants [9]. Pre-implant prostate volumes were measured by trans-rectal ultrasound, and post-procedure prostate size was calculated by serial CT scans on days 1 , $8,30,90$, and 180 . Their data showed that the majority of the edema resolved in the first week with very little volume decrease observed past day 30. Despite the differences in techniques used between this study and ours, the findings strongly support that the post-implant edema resolves within one month. Sophisticated bio-mathematical models have been developed based on both edema resolution pattern and the half-life of the isotope used for treatment $[10-12,17]$. These studies are based on dynamic changes in prostate volume and seed location during dose delivery. Based on these models, the optimum timing of CT dosimetry (to maintain the mean error below $5 \%$ ) for iodine- 125 is 5 to 9 weeks post-implantation. Dogan et al. did not recommend specific timing for CT dosimetry, but confirmed substantial improvement in prostate prescription dose coverage from $77 \%$ on day 1 to $85 \%$ on day 28 [13]. Tanaka et al. prospectively studied 74 patients undergoing I-125 prostate implant using CT/MRI fusion; dosimetry was performed on day 1 and day 30. Low quality implants from day 1 (with V100 of $<80 \%$ ) were associated with substantial edema which improved by day 30 [19]. Ohashi et al. compared intra-operative TRUS based dosimetry with actual day 1 and day 30 coverage and found no difference between intra-operative and day 30 target coverage [20]. While this may eliminate the need for post-operative CT-based
Table 4. Mean ratio of actual day 1 and day 30 values to estimated day 30 dosimetry and estimated day 30 dosimetry to actual day 30 dosimetry across all patients

\begin{tabular}{lcc} 
& Ratio & $\begin{array}{c}p \text {-value } \\
\text { (sign test) }\end{array}$ \\
\hline Actual $\mathrm{D}_{90}$ Day1/Actual $\mathrm{D}_{90}$ Day30 & 0.7117 & 0.0078 \\
\hline Estimated $\mathrm{D}_{90}$ Day30/Actual $\mathrm{D}_{90}$ Day30 & 0.9365 & 1.0000
\end{tabular}

${ }^{*} p$ values were calculated for actual day 1 and estimated day 30 values as compared with the actual day 30 dosimetry

dosimetry in the future, a large proportion of centers have not yet adopted an intra-operative planning approach and, therefore, must continue to depend on post-implant CTbased dosimetry to evaluate the quality of their implants.

In addition to the resolution pattern of prostate edema, we analyzed the effect of several variables on the extent of the swelling. In contrast to previous reports, we found that those patients who had monotherapy, low Gleason score, and higher activity seeds experienced greater edema in the prostate gland ( $p$ values $=0.02,0.046,0.02$ respectively) . When brachytherapy is used as a boost to EBRT, lower activity seeds $(0.3 \mathrm{mCi})$ are used. Patients who received lower activity seeds experienced on average $33 \%$ less swelling than patients in whom $0.5 \mathrm{mCi}$ seeds were used. These findings suggest that edema may not be simply a result of physical trauma to the gland, but may be affected by the radiation dose as well.

Further examination of seed strength as an independent variable may pose a challenge given its relationship to other factors. Patients with a low Gleason score and low risk overall receive monotherapy with high strength seeds; as such, it is difficult to assess seed strength, Gleason score, and monotherapy as individual factors. Of note, we did not appreciate a generalized trend suggesting that the Gleason score has an inverse relationship to the degree of edema. Our data showed that only the group with Gleason $\leq 6$ who received high dose seeds had statistically greater edema $(p=0.046)$. These conclusions may be limited by the conditions of our study. Our study's sample size does not provide the statistical power to fully validate these conclusions and may be strengthened on further patient accrual. Also, the choice of CT imaging to monitor change in prostate volume as opposed to MRI increases the likelihood of volume discrepancies. Given the prevalence of CT in treatment planning and dosimetry calculations, it is important to explore areas of improvement in our current techniques. Although we were able to eliminate inter-observer variability by having a single radiation oncologist contour all prostate volumes, we were unable to adequately address intra-observer variability, which could result in inaccurate prostate volumes.

Another area of concern is that edema may affect the dose volume calculations. Prostate dosimetry calculations can easily be underestimated when post-implant $\mathrm{CT}$ scans are performed prior to the resolution of prostate swelling. Similar to previous studies, we found that edema frequently has an adverse effect on implant dosimetry [23]. In the study by Waterman et al., edema affected post-implant dosimetry in 
seven of ten cases [8]. In Prestidge's study, the prostate volume covered by the $80 \%$ isodose line increased from $85.6 \%$ on postoperative day 1 to $92.2 \%$ on day 180 (9). In our study, patients had an average $D_{90}$ of $83 \%$ on day 1 as compared to day 30 . While the discrepancy between the day 1 and day 30 dosimetry was significant, our method of estimating the day 30 dosimetry using a CF yielded values that were an approximation of the actual $\mathrm{D}_{90}$. Comparing our estimates to actual dosimetry revealed a non-significant ratio, supporting this approximation (Table 4).

While there is consensus that edema in the immediate post-operative period negatively affects prostate coverage, it is often desirable to perform CT shortly after the procedure. This allows for faster feedback, but may result in underestimation of the prostate dose. When it is not possible for a patient to return for a day $30 \mathrm{scan}, \mathrm{D}_{90}$ Day 1 could be divided by a correction factor to more accurately reflect the quality of the implant.

\section{Conclusions}

We found that maximum prostate size is observed in the immediate post-operative period, returning to the preimplant volume by day 30 . There were several factors which we were able to identify as associated with greater prostate edema, namely low Gleason $(\leq 6)$ score, higher seed activity ( $\geq 4 \mathrm{mCi})$, and monotherapy use. We found a significant difference between day 1 and day 30 dosimetry; it was $83 \%$ on day 1 as compared to day 30 . This suggests that postimplant dosimetry should be obtained at least one month after implantation in order to obtain an accurate sense of implant quality. This could allow for resolution of swelling and avoids the consequential underestimation of gland coverage. In the event that such scheduling is not feasible, we found that day 30 dosimetry can be estimated by dividing $\mathrm{D}_{90}$ Day 1 by a volume correction factor of 0.76 . Further analysis of a larger cohort could validate a corrected $D_{90}$ value that more accurately reflects dose to the prostate than using day 1 or day 9 CT-based dosimetry.

\section{References}

1. Horner MJ, Ries LAG, Krapcho M et al. SEER Cancer Statistics Review, 1975-2006. National Cancer Institute, Bethesda; http://seer.cancer.gov/csr/1975_2006/ [2011, October 12].

2. Sylvester JE, Grimm PD, Blasko JC et al. 15-year biochemical relapse free survival in clinical Stage T1-T3 prostate cancer following combined external beam radiotherapy and brachytherapy; Seattle experience. Int J Radiat Oncol Biol Phys 2007; 67: 57-64.

3. Merrick GS, Butler WM, Dorsey AT et al. 5-year biochemical outcome following permanent interstitial brachytherapy for clinical T1-T3 prostate cancer. Int J Radiat Oncol Biol Phys 2001; 51: 41-48.

4. Kollmeier MA, Stock RG, Stone N. Biochemical outcomes after prostate brachytherapy with 5-year minimal follow-up: importance of patient selection and implant quality. Int J Radiat Oncol Biol Phys 2003; 57: 645-653.

5. Zelefsky MJ, Wallner KE, Ling CC et al. Comparison of the 5 -year outcome and morbidity of three-dimensional conformal radiotherapy versus transperineal permanent Iodine- 125 implantation for early-stage prostatic cancer. J Clin Oncol 1999; 17: 517-522.
6. National Comprehensive Cancer Network. NCCN Guidelines: Prostate Cancer; http:/ / www.nccn.org/professionals/physician_gls/PDF/prostate.pdf [2011, August 25].

7. Devlin PM. Brachytherapy Applications and Techniques. $1^{\text {st }}$ ed. Lippincott Williams \& Wilkins, Philadelphia 2007; pp. 181-218.

8. Waterman FM, Yue N, Corn BW et al. Edema associated with I-125 or Pd-103 prostate brachytherapy and its impact on postimplant dosimetry: an analysis based on serial CT acquisition. Int J Radiat Oncol Biol Phys 1998; 41: 1069-1077.

9. Prestidge BR, Bice WS, Kiefer EJ et al. Timing of computed tomography-based postimplant assessment following permanent transperineal prostate brachytherapy. Int J Radiat Oncol Biol Phys 1998; 40: 1111-1115.

10. Yue N, Dicker AP, Corn BW et al. A dynamic model for the estimation of optimum timing of computed tomography scan for dose evaluation of I-125 or Pd-103 seed implant of the prostate. Int J Radiat Oncol Biol Phys 1999; 43: 447-454.

11. Badiozamani KR, Wallner K, Sutlief $S$ et al. Anticipating prostatic volume changes due to prostate brachytherapy. Radiat Oncol Investig 1999; 4: 360-364.

12. Yue N, Chen Z, Peschel R et al. Optimum timing for imagebased dose evaluation of I-125 and Pd-103 prostate seed implants. Int J Radiat Oncol Biol Phys 1999; 45: 1063-1072.

13. Dogan N, Mohideen N, Glasgow G et al. Effect of prostatic edema on CT-based postimplant dosimetry. Int J Radiat Oncol Biol Phys 2002; 53: 483-489.

14. Crook J, McLean M, Yeung I et al. MRI-CT fusion to assess postbrachytherapy prostate volume and the effects of prolonged edema on dosimetry following transperineal interstitial permanent prostate brachytherapy. Brachytherapy 2004; 3: 55-60.

15. Reed DR, Wallner K, Ford E et al. Effect of post-implant edema on prostate brachytherapy treatment margins. Int J Radiat Oncol Biol Phys 2005; 63: 1469-1473.

16. Pinkawa M, Gagel B, Piroth MD et al. Changes of dose delivery distribution within the first month after permanent interstitial brachytherapy for prostate Cancer. Strahlenther Onkol 2006; 182: 525-530.

17. Leclerc G, Lavallee MC, Roy R et al. Prostatic edema in I-125 permanent prostate implants: Dynamical dosimetry taking volume changes into account. Med Phys 2006; 33: 574-583.

18. Yue N, Mori J, Nath R et al. External beam radiotherapy boosts to reduce the impact caused by edema in prostate permanent seed implants. Phys Med Biol 2006; 51: 2267-2277.

19. Tanaka O, Hayashi S, Matsuo M et al. Effect of edema on postimplant dosimetry in prostate brachytherapy using CT/MRI fusion. Int J Radiat Oncol Biol Phys 2007; 69: 614-618.

20. Ohashi T, Yorozu A, Toya K et al. Comparison of intraoperative ultrasound with postimplant computed tomography-dosimetric values at Day 1 and Day 30 after prostate brachytherapy. Brachytherapy 2007; 6: 246-253.

21. Erickson B. Image-Based Brachytherapy: A Forum for collaboration between radiation oncologists and diagnostic radiologists. J Am Coll of Radiol 2005; 2: 753-758.

22. Nath R, Bice WS, Butler WM et al. AAPM recommendations on dose prescription and reporting methods for permanent interstitial brachytherapy for prostate cancer: report of Task Group 137. Med Phys 2009; 36: 5310-5322

23. Knaup C, Mavroidis P, Stathakis S et al. Evaluation of the effect of prostate volume change on tumor control probability in LDR brachytherapy. J Contemp Brachyther 2011; 3: 125-130. 